\title{
Improve Quality of Service (QOS) Using Equalization Techniques
}

\author{
Al-Zayadi Haider ${ }^{1}$, Samer Salah Thabit ${ }^{2}$ \\ RAED Yahya Abdulghafoor
}

\begin{abstract}
Bit error rate in mobile communication is of parameters which effect on performance of network then effect on service quality of network. There are many technique used to improve this performance one of these techniques are equalization. As we know there are many type of equalization here in our article we will use zero forcing equalizer (ZF) and zero forcing with successive interference cancellation(ZF-SIC) which technique used to improving zero forcing equalization in another meaning used this technique to combat Inter-symbol interference (ISI) in network as our goal to improve quality of service (Qos).
\end{abstract}

Keyword: MIMO, ZF, SIC, Bit error rate.

\section{Introduction}

Orthogonal Frequency Division Multiplexing (OFDM) is one of the promising technologies to mitigate the ISI. In an OFDM signal the bandwidth is divided into many narrow sub-channels which are transmitted in parallel. Each subchannel is typically chosen narrow enough to eliminate the effect of delay spread. By combining OFDM with CDMA dispersive fading limitations of the cellular mobile radio environment can be overcome and the effects of co-channel interference can be reduced.As we know data transmission at high bit rates is essential for many services such as video, high quality audio and mobile integrated service digital network. When the data is transmitted at high bit rates, over mobile radio channels, the channel impulse response can extend over many symbol periods, which lead to Intersymbol interference (ISI). Inter-symbol Interference always causes an issue for signal recovery in wireless communication. This can be combated with application of an equalizer. Equalization compensates for Inter-symbol Interference (ISI) created by multipath signal prorogation within time dispersive channels.We considering 2 transmit2 receive antenna case (resulting in a $2 \times 2$ MIMO channel). Assume that the channel is a flat fading Rayleigh multipath channel and the modulation is BPSK. To achieve such an objective that mitigate ISI its need strong used equalization techniques .According to these point it lead to improve ZF equalizer by used ZF-SIC equalizer.

\section{MIMO System}

In radio, multiple-input and multiple-output, or MIMO is the use of multiple antennas at both the transmitter and receiver to improve communication performance. It is one of several forms of smart antenna technology. Note that the terms input and output refer to the radio channel carrying the signal, not to the devices having antennas.

MIMO technology has attracted attention in wireless communications, because it offers significant increases in data throughput and link range without additional bandwidth or increased transmit power. It achieves this goal by spreading the same total transmit power over the antennas to achieve an array gain that improves the spectral efficiency (more bits per second per hertz of bandwidth) and/or to achieve adversity that improves the link reliability (reduced fading). Because of these properties, MIMO is an important part of modern wireless communication which is clearly in figure 1 below.

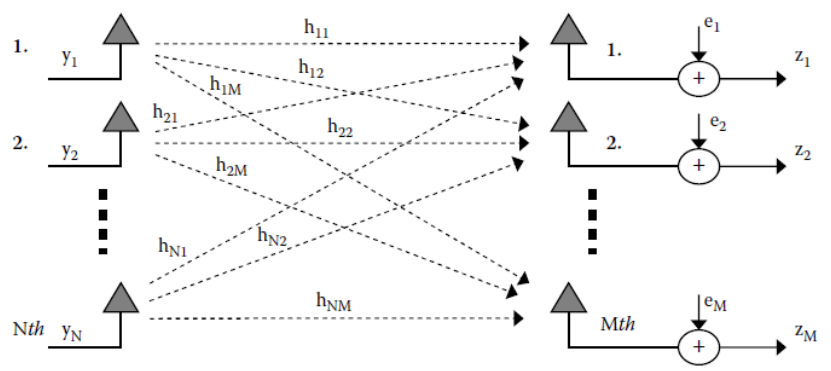

Figure 1: Multiple input multiple output system

\section{Functions of MIMO}

MIMO can be sub-divided into three main categories, precoding, spatial multiplexingor SM, and diversity coding. Precodingis multi-stream beam-forming, in the narrowest definition. In more general terms, it is considered to be all spatial processing that occurs at the transmitter. In (singlestream) beam-forming, the same signal is emitted from each of the transmit antennas with appropriate phase and gain weighting such that the signal power is maximized at the receiver input. The benefits of beam-forming are to increase the received signal gain, by making signals emitted from different antennas add up constructively, and to reduce the multipath fading effect. In line-of-sight propagation, beamforming results in a well-defined directional pattern. However, conventional beams are not a good analogy in cellular networks, which are mainly characterized by multipath propagation. When the receiver has multiple antennas, the transmit beam-forming cannot simultaneously maximize the signal level at all of the receive antennas, and precoding with multiple streams is often beneficial. Note that precoding requires knowledge of channel state information(CSI) at the transmitter and the receiver.

Spatial multiplexingre quires MIMO antenna configuration. In spatial multiplexing, a high rate signal is split into multiple lower rate streams and each stream is transmitted from a different transmit antenna in the same frequency 


\section{International Journal of Science and Research (IJSR) \\ ISSN (Online): 2319-7064 \\ Index Copernicus Value (2013): 6.14 | Impact Factor (2013): 4.438}

channel. If these signals arrive at the receiver antenna array with sufficiently different spatial signatures and the receiver has accurate CSI, it can separate these streams into (almost) parallel channels. Spatial multiplexing is a very powerful technique for increasing channel capacity at higher signal-tonoise ratios (SNR). The maximum number of spatial streams is limited by the lesser of the number of antennas at the transmitter or receiver. Spatial multiplexing can be used without CSI at the transmitter, but can be combined with proceed in gif CSI is available. Spatial multiplexing can also be used for simultaneous transmission to multiple receivers, known as space-division multiple access or multi-user MIMO, in which case CSI is required at the transmitter. The scheduling of receivers with different spatial signatures allows good separability.

Diversity Coding techniques are used when there is no channel knowledge at the transmitter. In diversity methods, a single stream (unlike multiple streams in spatial multiplexing) is transmitted, but the signal is coded using techniques called space-time coding. The signal is emitted from each of the transmit antennas with full or near orthogonal coding. Diversity coding exploits the independent fading in the multiple antenna links to enhance signal diversity. Because there is no channel knowledge, there is no beam-forming or array gainfrom diversity coding. Diversity coding can be combined with spatial multiplexing when some channel knowledge is available at the transmitter. In mathematical expression $N x M$ MIMO system, as depicted in Fig. 1.The input and the output should be interpreted from the radio channel perspective(i.e., the $N$ transmit antennas provide the input to the wireless channel, while the $M$ receive antennas deliver the output of the channel). The transfer function of the multi antenna system can be characterized by the channel transfer matrix $\mathbf{H}$. Where the $\mathrm{h}_{\mathrm{i}}$, jelement of the matrix gives the channel coefficient between transmit antenna $i$ and receive antenna $j$. This means that the system can be described by the following equation:

$$
\mathbf{z}=\mathbf{H} \cdot \mathbf{y}+\mathbf{e}
$$

That is,

$$
\left[\begin{array}{c}
z_{1} \\
\vdots \\
z_{M}
\end{array}\right]=\left[\begin{array}{cccc}
h_{1,1} & h_{1,2} & \ldots & h_{1, N} \\
h_{2,1} & h_{2,2} & \ldots & h_{2, N} \\
\vdots & \vdots & \ddots & \vdots \\
h_{M, 1} & h_{M, 2} & \ldots & h_{M, N}
\end{array}\right]\left[\begin{array}{c}
y_{1} \\
\vdots \\
y_{N}
\end{array}\right]+\left[\begin{array}{c}
e_{1} \\
\vdots \\
e_{M}
\end{array}\right]
$$

\section{Types of Equalizer}

We can classify equalization techniques into

1. Linear equalizer

2. NoN-Linear equalizer

- Decision Feedback Equalization (DFE)

- Maximum Likelihood symbol Detection

- Maximum Likelihood Sequence Estimation (MLSE)

3. Algorithms for Adaptive Equalizer

- Zero Forcing Algorithm

- Recursive Least Square Algorithm

- Least Mean Squares Algorithm (LMS)

These are classified base on how the output an adaptive in used for subsequent of the equalizer. According to figure 2 below we can understand what mean linear equalizer and non-linear equalizer .In linear equalization the output signal $\mathrm{d}(\mathrm{t})$ is not used in feedback path to adapt the equalizer while in no-linear equalization the output signal $d(t)$ is feedback to change the subsequent output of the equalizer [1].

An equalizer is usually implemented at the baseband or at IF in a receiver. Here we talk apart how related with equalizer which mean the signal received by the equalizer is given by

$$
\mathrm{Z}(\mathrm{t})=\mathrm{x}(\mathrm{t}) \otimes \mathrm{f}^{*}(\mathrm{t})+\mathrm{n}_{\mathrm{b}}(\mathrm{t})
$$

Where $\mathrm{x}(\mathrm{t})=$ original signal

$\mathrm{f}(\mathrm{t})=$ combined impulse response

$\mathrm{n}_{\mathrm{b}}(\mathrm{t})=$ base band noise

If the impulse response of the equalizer is $E_{e q}(t)$, the output of the equalizer is

$$
=h(t) \otimes g(t)+n_{b}(t) \otimes E_{e q}(t)
$$

The desired output is $x(t)$ which is the original source data assume that $\mathrm{n}_{\mathrm{b}}(\mathrm{t})=0$ then, in order that $\mathrm{d}(\mathrm{t})=\mathrm{x}(\mathrm{t})$ it must

$$
\mathrm{g}(\mathrm{t})=\mathrm{f}^{*}(\mathrm{t}) \otimes \mathrm{E}_{\text {eq }}(\mathrm{t})=\delta(\mathrm{t})
$$

The main goal of equalization is to satisfy the above equation in frequency domain it is given by

$$
E_{\text {eq }}(f) F(-f)=1
$$

Since the baseband complex envelope expression can be used to represent band pass waveforms, the channel response, demodulated signal and adaptive equalizer. Algorithms are usually simulated and implemented at the baseband.

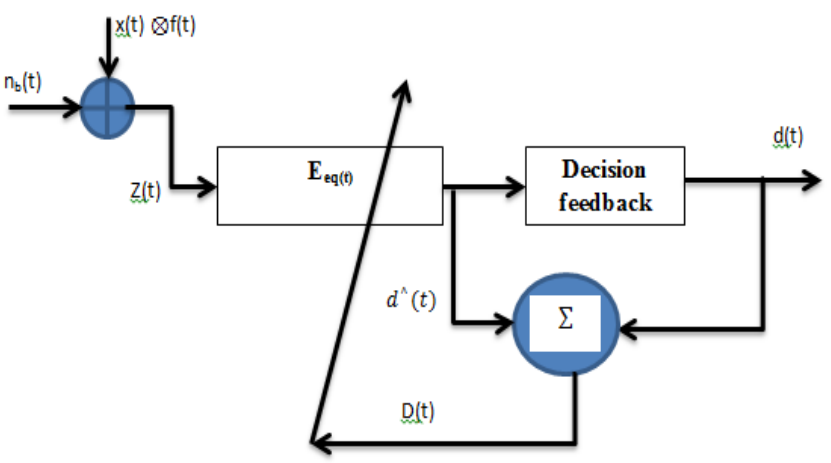

Figure 2: Block diagram of a communication system with anAdaptive equalizer in the receiver

So that the combination of the transmitter, channel, and receiver appear to be an all pass channel [2]. Analysis \& investigate the performance characteristics of two types of equalizers namely, ZF and ZF-SIC equalizers for MIMO wireless receiver.

\section{Zero Forcing Equalizer}

The Zero-Forcing algorithm (ZF) is based on detecting the components of $\mathrm{x}$ one by one. To solve for $\mathbf{X}$, know need to find a matrixW which satisfies WH=I. The Zero Forcing (ZF) linear detector for meeting this constraint is given by,

$$
\mathrm{W}=\left(\mathrm{H}^{\mathrm{H}} \mathrm{H}+\mathrm{N}_{\mathrm{o}} \mathrm{I}\right)^{-1} \mathrm{H}^{\mathrm{H}}
$$

This matrix is also known as the pseudo inverse for a general $\mathrm{m} \times \mathrm{n}$ matrix. The term,

$$
\mathrm{H}^{\mathrm{H}} \mathrm{H}=\left(\begin{array}{ll}
\mathrm{h}_{1,1}^{*} & \mathrm{~h}_{2,1}^{*} \\
\mathrm{~h}_{1,2}^{*} & \mathrm{~h}_{2,2}^{*}
\end{array}\right)\left(\begin{array}{ll}
\mathrm{h}_{1,1} & \mathrm{~h}_{1,2} \\
\mathrm{~h}_{2,1} & \mathrm{~h}_{2,2}
\end{array}\right)
$$

Note that the off diagonal terms in the matrix $\mathrm{H}^{\mathrm{H}} \mathrm{H}$ are not zero. Because the off diagonal terms are not zero, the zero forcing equalizer tries to null out the interfering terms when 


\section{International Journal of Science and Research (IJSR) \\ ISSN (Online): 2319-7064 \\ Index Copernicus Value (2013): 6.14 | Impact Factor (2013): 4.438}

performing the equalization, i.e. when solving for $\mathrm{x} 1$ the interference from $\mathrm{x} 2$ is tried to be nulled and vice versa.

While doing so, there can be amplification of noise. Hence Zero Forcing equalizer is not the best possible equalizer to do the job. However, it is simple and reasonably easy to implement [3].Obviously, incorrect symbol detection in the early stages will create errors in the following stages; i.e. error propagation. This is a severe problem with cancellation based detection techniques particularly. When the number of transmit and receive antennas are the same. The first detected symbol's performance is quite poor as it has no diversity. To reduce the effect of error propagation and to optimize the performance technique, it has been shown in that the order of detection can increase the performance considerably. By detecting the symbols with largest channel coefficient magnitude first, the effect of the noise vector producing an incorrect symbol can be reduced, and reducing error propagation as result.

\section{Successive Interference Analysis (SIC)}

Before understand ZF-SIC it must analysis SIC. In classical Successive Interference Cancellation, the receiver arbitrarily takes one of the estimated symbols (for example the symbol transmitted in the second spatial dimension, $\hat{x}_{2}$ ), and subtract its effect from the received symbol $y_{1}$ and $y_{2}$. Once the effect of $\hat{x}_{2}$ is removed, the new channel becomes a one transmit antenna, 2 receive antenna case and can be optimally equalized by Maximal Ratio Combining (MRC).

However, we can have more intelligence in choosing whether we should subtract the effect of $\hat{x}_{1}$ first or $\hat{x}_{2}$ first. To make that decision, let us find out the transmit symbol (after multiplication with the channel) which came at higher powerat the receiver. The received power at the both the antennas corresponding to the transmitted symbol $\mathrm{x}_{1}$ is,

$$
P x_{1}=\left|h_{1,1}\right|^{2}+\left|h_{2,1}\right|^{2}
$$

The received power at the both the antennas corresponding to the transmitted symbol $x_{2}$ is,

$$
P x_{2}=\left|h_{1,2}\right|^{2}+\left|h_{2,2}\right|^{2}
$$

If $\mathrm{Px}_{2}>\mathrm{Px}_{1}$ then the receiver decides to remove the effect of $\hat{x}_{1}$ from the received vector $\mathrm{y}_{1}$ and $\mathrm{y}_{2}$. Else if $\mathrm{Px}_{2} \leq \mathrm{Px}_{1}$ the receiver decides to subtract effect of $\hat{x}_{2}$ from thereceived vector $\mathrm{y}_{1}$ and $\mathrm{y}_{2}$, and then re-estimate $\hat{x}_{1}$. Once the effect of either $\hat{x}_{1}$ or $\hat{x}_{2}$ is removed, the new channel becomes a one transmit antenna, 2 receive antenna case and the symbol on the other spatial dimension can be optimally equalized by Maximal Ratio Combining (MRC).For detailed equations on the construction of the new $2 \times 1$ channel using successive interference cancellation, please refer to the post on ZF-SIC with optimal ordering [4].

\section{Zero Forcing Equalization with Successive Interference Cancellation (ZF-SIC)}

Successive interference cancellation (SIC) is a physical layer capability that allows a receiver to decode packets that arrive simultaneously. Since SIC is known to be sensitive to error propagation a careful adjustment of the data rate at each spatial layer is mandatory and decoding of already detected spatial layers is required prior to an interference cancelation step. Using the Zero Forcing (ZF) equalization approach described above, the receiver can obtain an estimate of the two transmitted symbols $\mathrm{x}_{1}$, $\mathrm{x}_{2}$, i.e.

$$
\left[\begin{array}{l}
\hat{x}_{1} \\
\hat{x}_{2}
\end{array}\right]=\left(H^{H} H\right)^{-1} H^{H}\left[\begin{array}{l}
y_{1} \\
y_{2}
\end{array}\right]
$$

Take one of the estimated symbols (for example $\hat{x}_{2}$ ) and subtract its effect from the received vector $\mathrm{y}_{1}$ and $\mathrm{y}_{2}$, i.e.

$$
\left[\begin{array}{l}
r_{1} \\
r_{2}
\end{array}\right]=\left[\begin{array}{l}
y_{1}-h_{1,2} \hat{x}_{2} \\
y_{2}-h_{2,2} \hat{x}_{2}
\end{array}\right]=\left[\begin{array}{l}
h_{1,1} x_{1}+n_{1} \\
h_{2,1} x_{1}+n_{2}
\end{array}\right]
$$

Expressing in matrix notation

$$
\begin{aligned}
& {\left[\begin{array}{l}
r_{1} \\
r_{2}
\end{array}\right]=\left[\begin{array}{l}
h_{1,1} \\
h_{2,1}
\end{array}\right] x_{1}+\left[\begin{array}{l}
n_{1} \\
n_{2}
\end{array}\right]} \\
& \mathrm{r}=\mathrm{hx}_{1}+\mathrm{n}
\end{aligned}
$$

The above equation is same as equation obtained for receive diversity case. Optimal way of combining the information from multiple copies of the received symbols in receive diversity case is to apply Maximal Ratio Combining (MRC).The equalized symbol is,

Where

$$
\hat{x}_{1}=\frac{h^{H} r}{h^{H} h}
$$

$$
\mathrm{h}^{\mathrm{H}} \mathrm{h}=\sum_{\mathrm{i}=1}^{\mathrm{N}}\left|\mathrm{h}_{\mathrm{i}}\right|^{2}
$$

i.e. sum of the channel powers across all the receive antennas. This forms the simple explanation for Zero Forcing Equalizer with Successive Interference Cancellation (ZFSIC) approach.

\section{Simulation}

The simulated results with a $2 \times 2$ MIMO system using BPSK modulation in Rayleigh channel is showing matching results. The zero forcing equalizer helps us to achieve the data rate gain, but not take advantage of diversity gain [5]. It can observe that in MIMO zero forcing in bit error rate if take 5 $\mathrm{dB}$ as average it can reach for bit error rate near 0.0638 in $2 \times 2$ MIMO zero forcing it clearly in figure 3 .

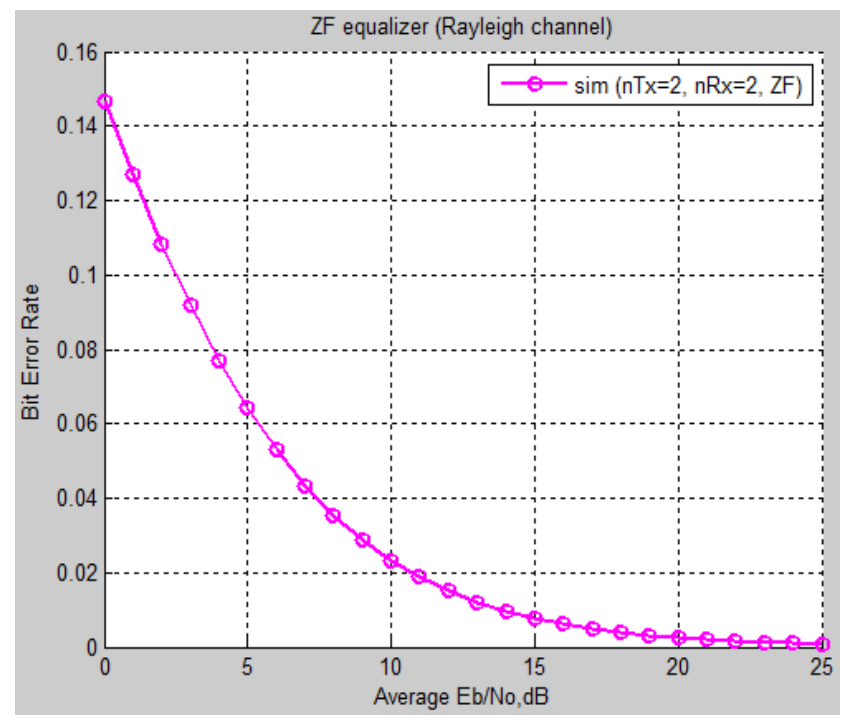

Figure 3: Zero Forcing Equalization (Rayleigh channel)

While an equalizer for on the SIC and the linear ZFenhaved is proposed. In each iteration, the proposed equalizer detects 


\section{International Journal of Science and Research (IJSR) \\ ISSN (Online): 2319-7064 \\ Index Copernicus Value (2013): 6.14 | Impact Factor (2013): 4.438}

and cancels a group of elements of the source vector in a receive signal. This SIC process allows the finite impulse response equalizers designed by SIC-ZFE as enhanced algorithm to be further optimized without error propagation problem. As Compared to Zero Forcing equalization alone case, addition of successive interference cancellation results in around $5 \mathrm{~dB}$ of improvement for BER of 0.044 as shown in figure 4 .

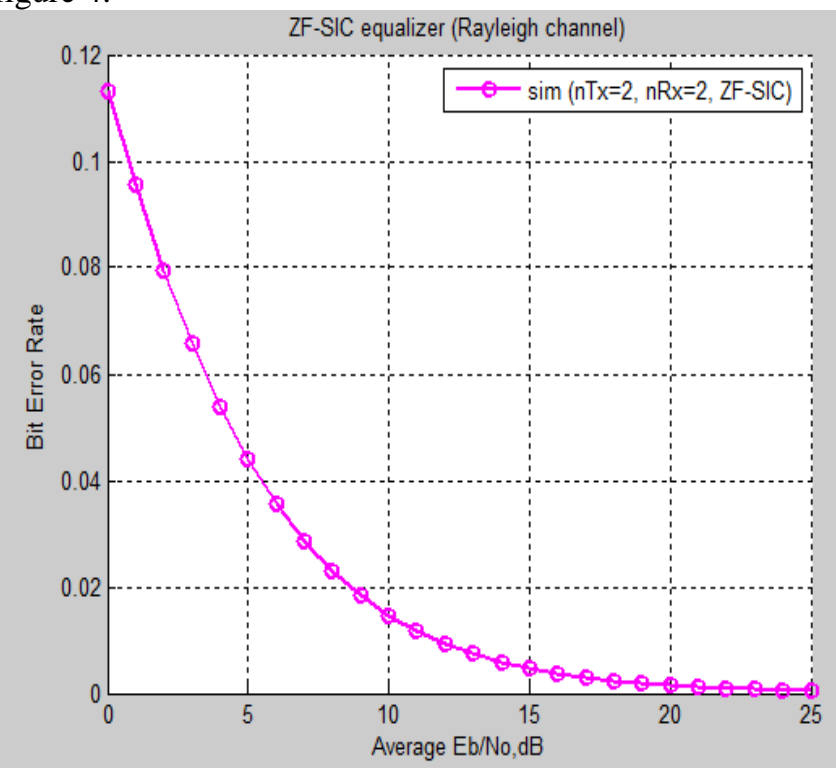

Figure 4: Zero Forcing Equalization with Successive Interference Cancellation (ZF-SIC)

The improvement is brought in because decoding of the information from the first spatial dimension (x1) has a lower error probability that the symbol transmitted from the second dimension. From figure 5[1]. After collect these tow techniques it clearly improved in bit error for ZF-SIC compare with ZF.It mean when used strong equalizer lead to got high quality of service (Qos) by means improve bit error rate.

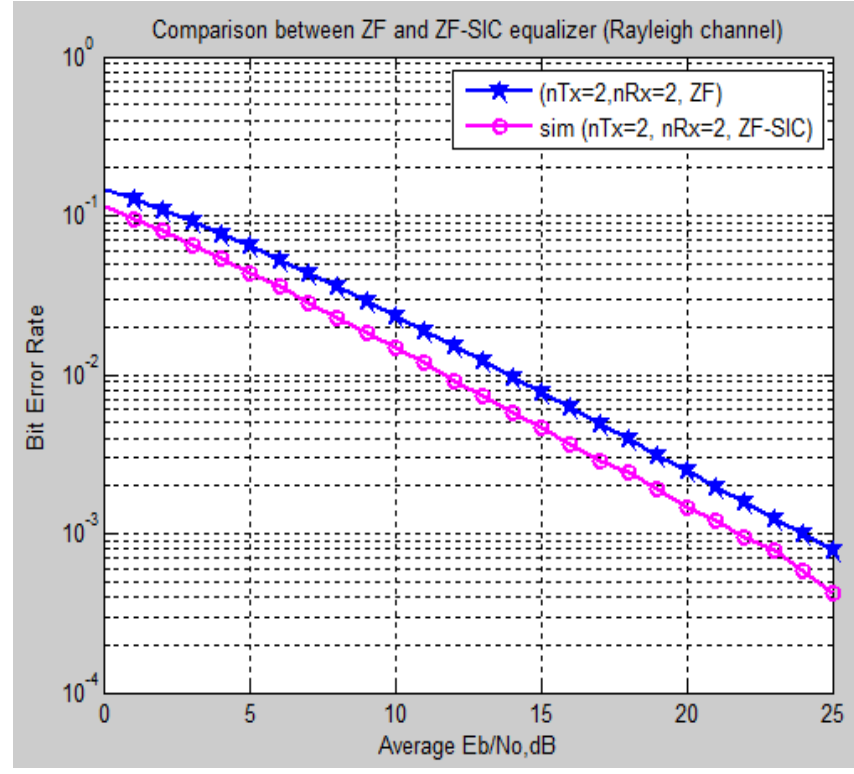

Figure 5: Combined ZF with ZF-SIC

\section{Conclusion}

Channel equalizer implementation becomes increasingly complex as data rates increase. Symbol times become shorter and receiver sample clocks must become correspondingly faster. ISI becomes much more severe-possibly spanning several symbol periods. The finite impulse response transversal filter is a common equalizer topology. As the period of the receiver sample clock $(\tau)$ decreases, more samples are required to compensate for a given amount of delay spread. Which mean number of delay taps increases along with the speed and complexity of the adaptive algorithm in LTE as ex. data rates(up to $100 \mathrm{Mbps}$ ) and delay spreads (approaching $17 \mu \mathrm{sec}$ ), this approach to channel equalization becomes impractical. As we know OFDM eliminates ISI in the time domain, which dramatically simplifies the task of channel compensation.. ZF-SIC improves the system performance as it compares then next or upcoming symbol with the previous received symbol and also offers low error probability compares to that $\mathrm{ZF}$. According to these results it can conclude more far that mean when reduce bit error rate lead to improve throughput network as we know that QoS one of parameters from throughput it mean so improve QoS.

\section{References}

[1]Al-Zayadi Haider. IMPROVE BIT ERROR RATE USING EQUALIZATION TECHNIQUES TCSET'2014 CONFERENCE PROGRAMME XII ${ }^{\text {th }}$ International Conference Dedicated to the 170th anniversary of the Lviv Polytechnic National University "MODERN PROBLEMS OF RADIO ENGINEERING, TELECOMMUNICATIONS, AND COMPUTER SCIENCE". Lviv, Ukraine 26 February-01 March 2014.Page No. 516-518.

[2] I. E. Telatar, "Capacity of multi-antenna Gaussian [channels," Eur. Trans.Tel., vol. 10, pp. 585-595, NovDec. 99"Zero-forcing equalization for time-varying systems with

[3] memory " by Cassio B. Ribeiro, Marcello L. R. de Campos, and Paulo S. R. Diniz

[4] R. Scholtz, "Multiple Access with Time-Hoping Impulse Modulaton," IEEE milit. Commun. Conf., vol . 2, pp. 447-450,1993.

[5] Approximate Minimum BER Power Allocation for MIMO Spatial Multiplexing Systems Neng Wang and Steven D. Blostein, Senior Member, IEEE 